\title{
What Investigators Need to Know About the Use of Animals
}

\author{
"Proper use of animals, including the avoidance or minimization of discomfort, \\ distress, and pain when consistent with sound scientific practices, is imperative." \\ U.S. Government Principle IV, 1985
}

Principal Investigators are responsible for the scientific and technical aspects of a grant award and must ensure compliance with Public Health Service (PHS) Policy on Humane Care and Use of Laboratory Animals (Policy) when using live vertebrate animals. PHS Policy incorporates the U.S. Government Principles for the Utilization and Care of Vertebrate Animals Used in Testing, Research, and Training, the Guide for the Care and Use of Laboratory Animals, and the American Veterinary Medical Association (AVMA) Guidelines for the Euthanasia of Animals. Vertebrate animals include traditional laboratory animals, farm animals, wildlife, and aquatic animals. Animal use encompasses research, teaching, or testing. Generation of custom antibodies is considered an activity involving vertebrate animals.

\section{Who Must Comply With the PHS Policy?}

The PHS Policy applies to extramural and intramural activities supported by any PHS agency, including the National Institutes of Health (NIH), the Food and Drug Administration, the Centers for Disease Control and Prevention, and the Department of Health and Human Services. All funding mechanisms, including research and training grants, cooperative agreements, and contracts, conducted at domestic and foreign institutions, are covered by the Policy. Additionally, activities conducted or supported by the Department of Veterans Affairs, the National Aeronautics and Space Administration, and the National Science Foundation are covered by the Policy under memoranda of understanding by these agencies with NIH.

\section{Good animal care and good science go hand in hand.}

\section{What is an IACUC?}

Institutional Animal Care and Use Committees (IACUCs) are local institutional committees with federally mandated oversight responsibilities, including:

- Reviewing animal-use protocols;

- Reviewing significant changes to protocols;

- Evaluating institutional compliance with PHS Policy, U.S. Department of Agriculture (USDA) Animal Welfare Act and Regulations, and institutional policies;

- Monitoring institutional animal care and use programs, including inspecting animal facilities;

- Reviewing concerns about animal care or use; and

- Reporting noncompliance and suspensions to the NIH Office of Laboratory Animal Welfare (OLAW).

\section{Investigator Responsibilities}

Investigators are accountable for the protection of the research animals in their care from the earliest stages of planning until a study is completed, including:

- Describing proposed use of animals in grant applications;

- Obtaining IACUC approval prior to using animals and prior to implementing significant changes;

- Ensuring research is conducted according to the protocol;

- Complying with institutional policies and procedures;

- Addressing significant changes to the use of animals in progress reports; and

- Addressing changes in the use of animals that may be a potential change in scope, including changes in performance site.

\section{Applying for Funding}

The proposed involvement of vertebrate animals is evaluated as part of the peer review process. Applicants must provide the following in grant applications:

1. Concise description of the proposed procedures to be used that involve vertebrate animals, including species, strains, ages, sex, and total number of animals by species to be used.

2. Justification that the species are appropriate for the proposed research and explanation why research goals can't be accomplished using an alternative model.

3. Description of the interventions including analgesia, anesthesia, sedation, palliative care and humane endpoints to minimize discomfort, distress, pain and injury.

4. Indication if the euthanasia method is consistent with AVMA Guidelines and a description of the method and scientific justification if it is not. 


\section{Obtaining IACUC Approval}

IACUC approval is required prior to award except in rare circumstances. Your IACUC will require you to submit information about the care and use of animals on a protocol form. Most animal-use protocols require a description of the following elements:

- Research project;

- Rationale for animal use and consideration of alternatives;

- Justification for the choice of species and number of animals;

- Research procedures involving animals;

- Procedures to minimize pain and distress;

- Animal living conditions and veterinary care;

- Names and qualifications of personnel who will perform work with animals;

- Method of euthanasia; and

- Endpoint criteria.

The use of animals as described in the protocol approved by the IACUC must be congruent with the description in a grant application. Any modification required by the IACUC that significantly changes the animal activity must be submitted to the funding agency along with the IACUC approval date.

"Investigators and other personnel shall be appropriately qualified and experienced for conducting procedures on living animals..." U.S. Government Principle VIII, 1985

\section{Receiving an Award}

To receive an award the grantee institution and every performance site where animal work will be performed must have an Animal Welfare Assurance approved by OLAW. OLAW will contact an institution with specific instructions when an Assurance is required. An Interinstitutional Assurance is negotiated when the grantee does not have its own animal facilities or IACUC and the animal work will be performed at an institution with an Assurance. The Foreign Assurance is required for institutions outside the U.S.

SBIR/STTR investigators should be aware of the requirements in order to address them in a timely fashion so that the necessary Assurances are in place and grants can be awarded.

The date of IACUC approval is essential in order to receive an award and will be requested if not previously provided.

\section{How to Write an Application Involving Research Animals \\ https://www.niaid.nih.gov/grants-contracts/ research-vertebrate-animals}

\section{Post Award}

IACUC review is required at least every 3 years (annually if covered by USDA regulations). Significant changes in animal care and use are to be approved by the IACUC prior to implementation. Check with your IACUC to determine what constitutes a significant change.

Grantees must also obtain prior approval from NIH for changes in scope, direction, or other areas that constitute a significant change from the aims, objectives, or purposes of the approved project. The grantee must make the initial determination of the significance of the change and should consult with a Grants Management Officer as necessary.

Conducting research in the absence of IACUC approval or implementing a significant change without IACUC approval constitutes noncompliance.

Charges to grant awards to conduct animal activities are not permitted without an approved Assurance or IACUC approval (including failure to obtain IACUC approval or when a protocol is expired or suspended by the IACUC).

Investigators also must be aware of and comply with additional institutional policies that may be more restrictive.

Resources:
NIH Office of Laboratory Animal Welfare page
on the VAS
https://olaw.nih.gov/guidance/vertebrate-animal-
section.htm
Guide for the Care and Use of Laboratory
Animals: Eighth Edition
https://olaw.nih.gov/sites/default/files/Guide-for-the-
Care-and-Use-of-Laboratory-Animals.pdf
NIH Grants Policy Statement
http://grants.nih.gov/policy/nihgps/index.htm
PHS 398 Grant Application
http://grants.nih.gov/grants/funding/phs398/phs398.html
How to Apply - Application Guidelines
https://grants.nih.gov/grants/how-to-apply-application-
guide.html
Vertebrate Animals Section Worksheet
http://grants.nih.gov/grants/olaw/VASchecklist.pdf
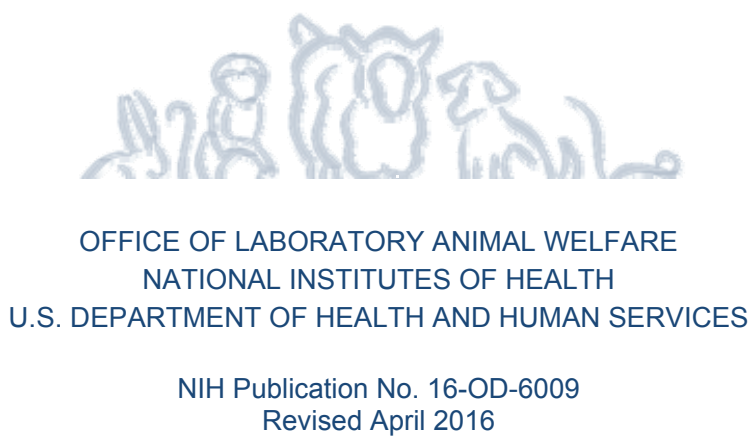

OFFICE OF LABORATORY ANIMAL WELFARE NATIONAL INSTITUTES OF HEALTH

NIH Publication No. 16-OD-6009 Revised April 2016 